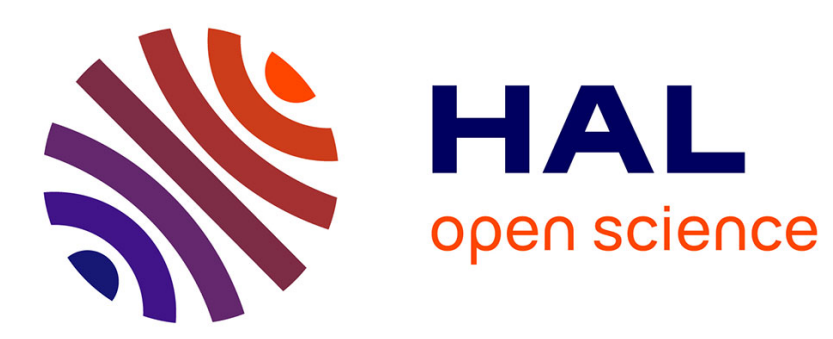

\title{
Friction force studies on layered materials using an Atomic Force Microscope
}

\author{
Hubert Klein, D. Pailharey, Y. Mathey
}

\section{To cite this version:}

Hubert Klein, D. Pailharey, Y. Mathey. Friction force studies on layered materials using an Atomic Force Microscope. Surface Science: A Journal Devoted to the Physics and Chemistry of Interfaces, 1997, 387 (1-3), pp.227-235. 10.1016/S0039-6028(97)00358-0 . hal-01770306

\section{HAL Id: hal-01770306 https://hal-amu.archives-ouvertes.fr/hal-01770306}

Submitted on 4 May 2018

HAL is a multi-disciplinary open access archive for the deposit and dissemination of scientific research documents, whether they are published or not. The documents may come from teaching and research institutions in France or abroad, or from public or private research centers.
L'archive ouverte pluridisciplinaire HAL, est destinée au dépôt et à la diffusion de documents scientifiques de niveau recherche, publiés ou non, émanant des établissements d'enseignement et de recherche français ou étrangers, des laboratoires publics ou privés. 


\title{
Friction force studies on layered materials using an Atomic Force Microscope
}

\author{
H. Klein *, D. Pailharey, Y. Mathey \\ Groupe de Physique des Etats Condensés UMR 6631 CNRS, case 901, Université de la Méditerranée, 13288 Marseille, Cedex 9, France
}

\begin{abstract}
We have studied friction force versus normal load on the nanometer scale on three layered materials which are expected to exhibit different mechanical properties: $2 \mathrm{H}-\mathrm{MoS}_{2}, 2 \mathrm{H}-\mathrm{NbSe}_{2}, \alpha-\mathrm{MoO}_{3}$. Different behaviours have been observed, such as plastic deformation and local wear. Under well-defined conditions there exists an analogy between nanoscopic experimental results and macroscopic friction results predicted by models (Amontons' law for instance). It is then possible to extract mean values of the friction coefficient on the nanometer scale. We are interested in friction measurements under atmospheric conditions which correspond to real sliding conditions. The influence of parameters such as surface pollution and scan speed appears to be determinant for the reliability of the results.
\end{abstract}

Keywords: Atomic force microscopy; Friction; Single crystal surfaces; Tribology

\section{Introduction}

Atomic force microscopy (AFM) is known as a convenient tool for imaging surfaces up to atomic resolution. Thanks to its conception, AFM is also an excellent tool for probing fundamental forces, Van der Waals and electrostatic forces for instance, and more particularly friction forces. Recently, AFM has brought a new understanding in fundamental mechanisms of friction and adhesion on the atomic scale [1-6]. But it could also be powerful for the comprehension of friction phenomena (wear and degradation) on the nanometer scale [7], which is of great interest for industry. From this point of view, investigation on layered materi-

\footnotetext{
* Corresponding author. Tel: $(+33)$ 491.829.175; fax: $(+33)$ 491.829.176; e-mail: klein@gpec.univ-mrs.fr
}

als $[1,8,9]$ constitutes a model case for these problems, and it is of interest to perform friction studies on these prototype surfaces under atmospheric, i.e. real, conditions.

\section{Principles of friction force measurements using AFM}

By scanning the AFM probe (a sharp tip mounted on a flexible cantilever) over a surface, the interactions between the tip and the surface induce cantilever deflections recorded by deviation of a laser beam as shown in Fig. 1. These deflections contain both topographic and frictional information about the surface.

According to several models [10] (Amontons' 


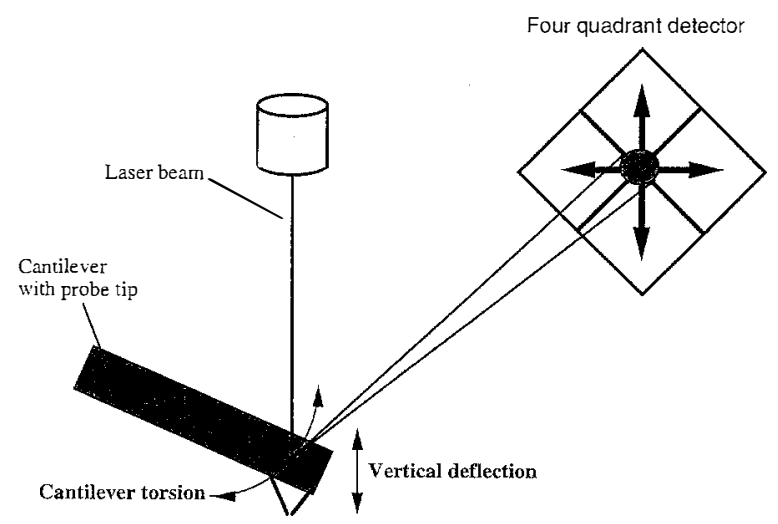

Fig. 1. Schematic description of the cantilever movement detection: tip displacements lead to movement of the laser beam reflected on the cantilever. This movement is detected on the four quadrant photodiode.

law, JKR model, etc.), the friction force $F_{\mathrm{f}}$ is dependent on the normal load $F_{\mathrm{n}}$.

In addition, on the nanometer scale the friction force intensity depends also on further parameters such as surface pollution $[11,12]$, which modifies the characteristics of the tip/surface interface, surface roughness [13], which causes normal load variations, or tilt of the probed surface out of the AFM scan plane [14], which leads to variation of the signal level read on the microscope detector.

\section{Experimental measurements of $F_{\mathrm{f}}$}

In AFM the intensity of the normal load $F_{\mathrm{n}}$ applied to the tip is easily obtained by multiplying the cantilever deflection $\Delta z$ by its normal spring constant $\Gamma_{\mathrm{z}}$ (in our case a V-shaped silicon nitride cantilever, $193 \mu \mathrm{m}$ in length, normal spring $\Gamma_{\mathrm{z}}=0.03 \mathrm{~N} \mathrm{~m}^{-1}$ ):

$F_{\mathrm{n}}=\Gamma_{\mathrm{z}} \cdot \Delta z$.

This normal load is in the range of a few $10^{-8} \mathrm{~N}$, and contains both the interfacial adhesive force and the externally applied force normal to the surface [15].

During lateral scanning (perpendicular to the longitudinal axis of the cantilever), cantilever torsion occurs due to friction force at the tip/surface interface. For a flat surface (i.e. atomically smooth), the detected signal depends mainly on the friction phenomenon (Fig. 2). Nevertheless, due to sample roughness, the lateral scan leads to a mixed signal where a component resulting from sample topography is superimposed on the real friction signal. Roughness can also affect local values of $F_{\mathrm{n}}$ and, consequently, the friction coefficient [13]. To minimize this phenomenon, it is necessary to investigate surfaces with the minimum of roughness and, when comparisons are desired, to compare surfaces with similar roughnesses. Following these requirements, cantilever torsions are mainly produced by the friction force resulting from the applied normal load and tip movement.

For such a lateral mode it could be tempting to use, in analogy with Eq. (1), a very simple relationship between cantilever torsion angle and friction force:

$F_{\mathrm{f}}=\Gamma_{\mathrm{t}} \cdot \theta$

where $\Gamma_{\mathrm{t}}$ represents the torsional spring constant of the cantilever, and $\theta$ the angle of torsion. Nevertheless, such a procedure is not trivial using a commercial atomic force microscope (Nanoscope III, Digital Instruments). Indeed, $\Gamma_{\mathrm{t}}$ depends only on cantilever and tip geometry and material constants $(E, G, v)$ of the cantilever [16]. This constant $\Gamma_{\mathrm{t}}$ could be obtained by calculation [17] or experimental calibration [18]. But the measurement of $\theta$ and consequently the measurement of $F_{\mathrm{f}}$ intensity is connected to the microscope setup (geometry,

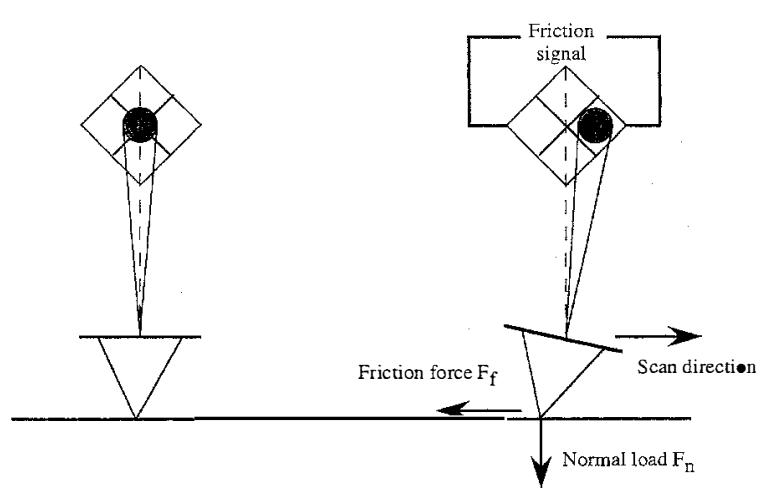

Fig. 2. Schematic representation of a tip submitted to a friction force during a lateral scan. 
sensitivity of the photodiodes). Therefore, it is clear that we need an accurate method to calibrate our $F_{\mathrm{f}}$ measurements and to extract friction contributions from the torsion signal detected during a lateral scan.

Ruan and Bhushan [19] have proposed an original method to calibrate the atomic force microscope for these measurements, using both usual (parallel to the longitudinal axis of the cantilever) and lateral scanning. In usual scanning, the atomic force microscope is already calibrated to measure vertical deflections of the cantilever and consequently the forces applied to the tip (Eq. (1)). In this direction, the friction force is superimposed on the normal force, and the measured signal contains both topographic and frictional information (Fig. 3). Therefore, upon operating on very flat surfaces where the topographic signal is negligible with respect to the friction signal, we can obtain meaningful $F_{\mathrm{f}}$ values. As we expect the same friction force at the tip/surface interface during a usual scan as during a lateral scan, we can link the friction signal $V_{\mathrm{f}}$ (in volts) detected upon laterally scanning to the values extracted from the usual scan. This method leads to a relationship:

$F_{\mathrm{f}}=k \cdot V_{\mathrm{f}}$.

However, to obtain an exact value of $k$ it is important to suppress the topographic contribution of the signal. The topographic signal is independent of scan direction, as opposed to the friction signal which has an algebraic intensity depending on the scan direction. By subtracting

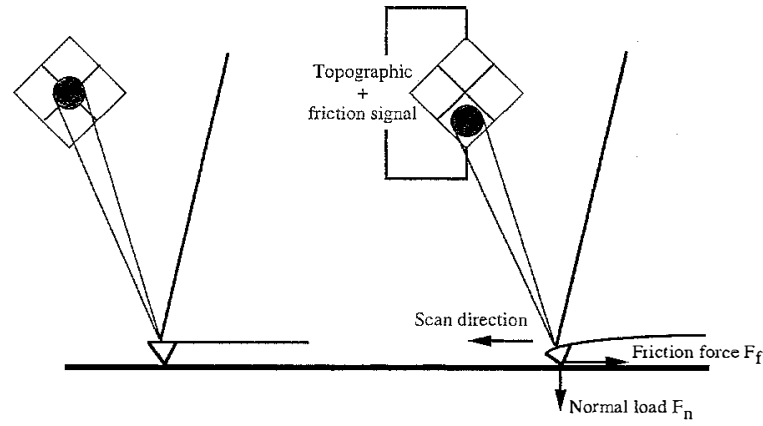

Fig. 3. Superimposition of topographic and frictional information during a usual scan. two AFM pictures recorded in the forward and backward scan directions, we could then obtain a mean value of the true friction signal.

In our case, the calibration carried out on a flat (111) silicon single crystal (less than $0.1 \mathrm{~nm}$ RMS) gives $k=2 \pm 0.2 \times 10^{-9} \mathrm{~N}^{-1}{ }^{1}$

\section{Different frictional behaviours for lamellar materials}

We study here three layered materials, $2 \mathrm{H}-\mathrm{MoS}_{2}, 2 \mathrm{H}-\mathrm{NbSe}_{2}$ and $\alpha-\mathrm{MoO}_{3}$ [20], which have their general bidimensional structure in common, but differ in their interlayer cohesion because of their electronic $\left(\mathrm{d}^{2}\right.$ Mo for $\mathrm{MoS}_{2}, \mathrm{~d}^{1}$ $\mathrm{Nb}$ for $\mathrm{NbSe}_{2}$ and $\mathrm{d}^{0} \mathrm{Mo}$ for $\mathrm{MoO}_{3}$ ) and crystallographic structures (hexagonal with different unit cell parameters for $\mathrm{MoS}_{2}$ and $\mathrm{NbSe}_{2}$, orthorhombic for $\mathrm{MoO}_{3}$ ). Upon considering Van der Waals gaps [21], $\mathrm{MoS}_{2}$ is expected to have the lowest and $\mathrm{MoO}_{3}$ the highest interlayer cohesion. For these reasons we expect different frictional behaviour for these three materials during AFM scans.

For our tests, samples are all single crystals freshly cleaved along their highest density plane. It is noteworthy that these surfaces are all atomically smooth at the beginning of the experiments (RMS in the range of $0.1 \mathrm{~nm}$ ). They are investigated by AFM in air, under the experimental conditions described above. All the experiments presented below are performed with the same tip ${ }^{2}$ on a $1 \mu \mathrm{m} \times 1 \mu \mathrm{m}$ square area at $1 \mu \mathrm{m} \mathrm{s}^{-1}$ scan speed. During the tests we make $F_{\mathbf{n}}$ variable, firstly increasing to a maximum value and then decreasing to the initial value, in order to put in evidence reversible and irreversible phenomena due to friction (wear for instance). We present hereafter graphs showing the variation of $F_{\mathrm{f}}$ vs. $F_{\mathrm{n}}$ for the three samples. We present also pictures recorded by AFM in topographic mode at the end of each friction acquisition series.

$2 \mathrm{H}-\mathrm{MoS}_{2}$ is known to be a good lubricant on

\footnotetext{
${ }^{1}$ NanoscopeIII AFM, Digital Instruments, V-shaped silicon nitride cantilever of $193 \mu \mathrm{m}$ length with $\Gamma_{\mathrm{z}}=0.03 \mathrm{~N} \mathrm{~m}^{-1}$.

${ }^{2} \mathrm{~V}$-shaped cantilever of $193 \mu \mathrm{m}$ length with $\Gamma_{\mathrm{z}}=0.03 \mathrm{~N} \mathrm{~m}^{-1}$.
} 
the macroscopic scale because of its low interlayer cohesion. Fig. 4(a) shows that there is no correlation between $F_{\mathrm{f}}$ and $F_{\mathrm{n}}$ following a known friction model, except in the first steps where $F_{\mathrm{f}}$ vs. $F_{\mathrm{n}}$ is more or less linear according to Amonton's law. ${ }^{3}$ Moreover, the topographic picture of Fig. 4(b) exhibits a surface where the investigated area during friction measurements is darker (meaning at a lower level) than the unaltered surface. The area corresponding to the presented results is delimited by a line, other darker areas correspond to preliminary experiments.

These facts lead us to think that the $\mathrm{MoS}_{2}$ surface has been modified during the experiment beyond a threshold value of $F_{\mathrm{n}}\left(2.3 \times 10^{-8} \mathrm{~N}\right)$. This modification could be explained by a plastic deformation (depression) of the material under the tip stress, because we have not seen on the surface profile (Fig. 5) any material rejected out of the investigated area.

In the case of $2 \mathrm{H}-\mathrm{NbSe}_{2}$, which has a higher interlayer cohesion than $\mathrm{MoS}_{2}$ (Fig. 6(a)), when $F_{\mathrm{n}}$ increases, $F_{\mathrm{f}}$ measurements are incoherent with large variations of intensity due to subsidiary phenomena. Interestingly enough, as soon as $F_{\mathrm{n}}$ decreases after the first normal loading, the relationship between $F_{\mathrm{f}}$ and $F_{\mathrm{n}}$ becomes linear, in full agreement with Amontons' law. After this first experiment we carried out several load/unload cycles showing a linear relationship $F_{\mathrm{f}}$ vs. $F_{\mathrm{n}}$, even if $F_{\mathrm{n}}$ is increasing again. The topographic picture after the first experiment (Fig. 6(b)) exhibits a surface with several holes in the investigated area (delimited by a line), and many material fragments scattered out of the investigated area during topographic picture recording on a larger size. The mean depth of the holes, in the range of $10 \AA$ (Fig. 7), roughly corresponds to the thickness (6.7 $\AA$ ) of one layer of $\mathrm{NbSe}_{2}$.

This clearly indicates a local wear during the first $F_{\mathrm{n}}$ increase, and after the sweeping away of layer fragments which disturb the microscope regulation, we obtain a linear relationship between $F_{\mathrm{f}}$ and $F_{\mathrm{n}}$ on a surface which remains unchanged with a constant RMS even if we perform further friction measurements with the same $F_{\mathrm{n}}$ range.

This phenomenon of wear on $\mathrm{NbSe}_{2}$ has been observed previously by Lieber and Kim [22].

For $\alpha-\mathrm{MoO}_{3}$, during the load/unload cycle of $F_{\text {n }}$, the relationship between $F_{\mathrm{f}}$ and $F_{\mathrm{n}}$ is linear

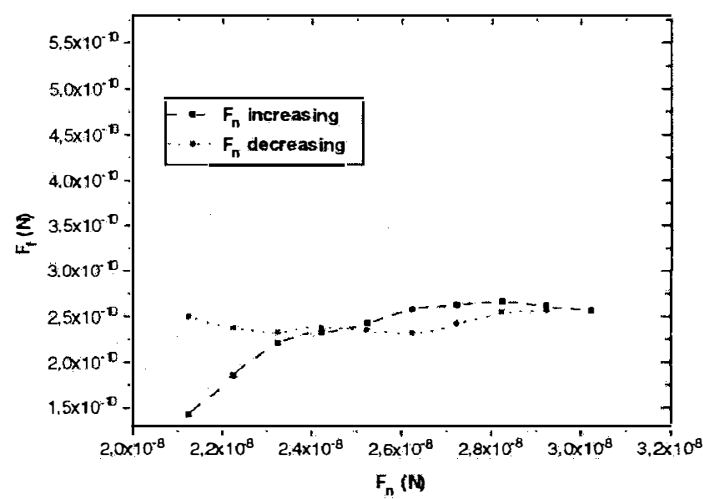

(a)

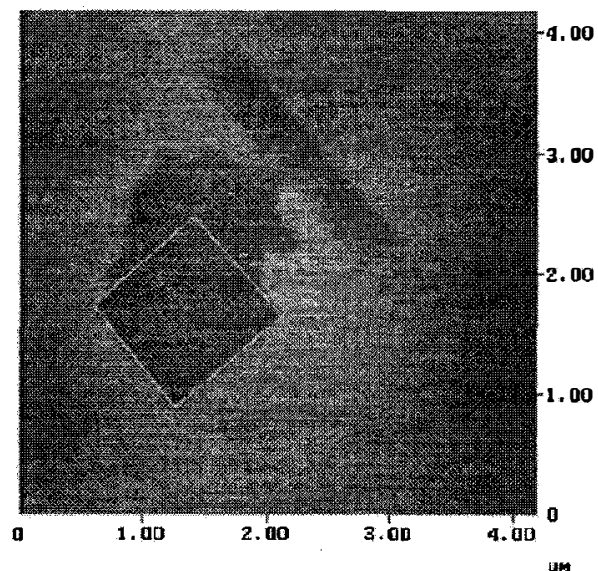

(b)

Fig. 4. (a) $F_{\mathrm{f}}$ vs. $F_{\mathrm{n}}$ during friction test on a $2 \mathrm{H}-\mathrm{MoS}_{2}$ single crystal. (b) AFM picture recorded after the friction test, exhibiting a depression of the surface.

\footnotetext{
${ }^{3}$ Amontons' law defines a constant coefficient of friction $\mu=F_{\mathrm{f}} / F_{\mathrm{n}}$.
} 

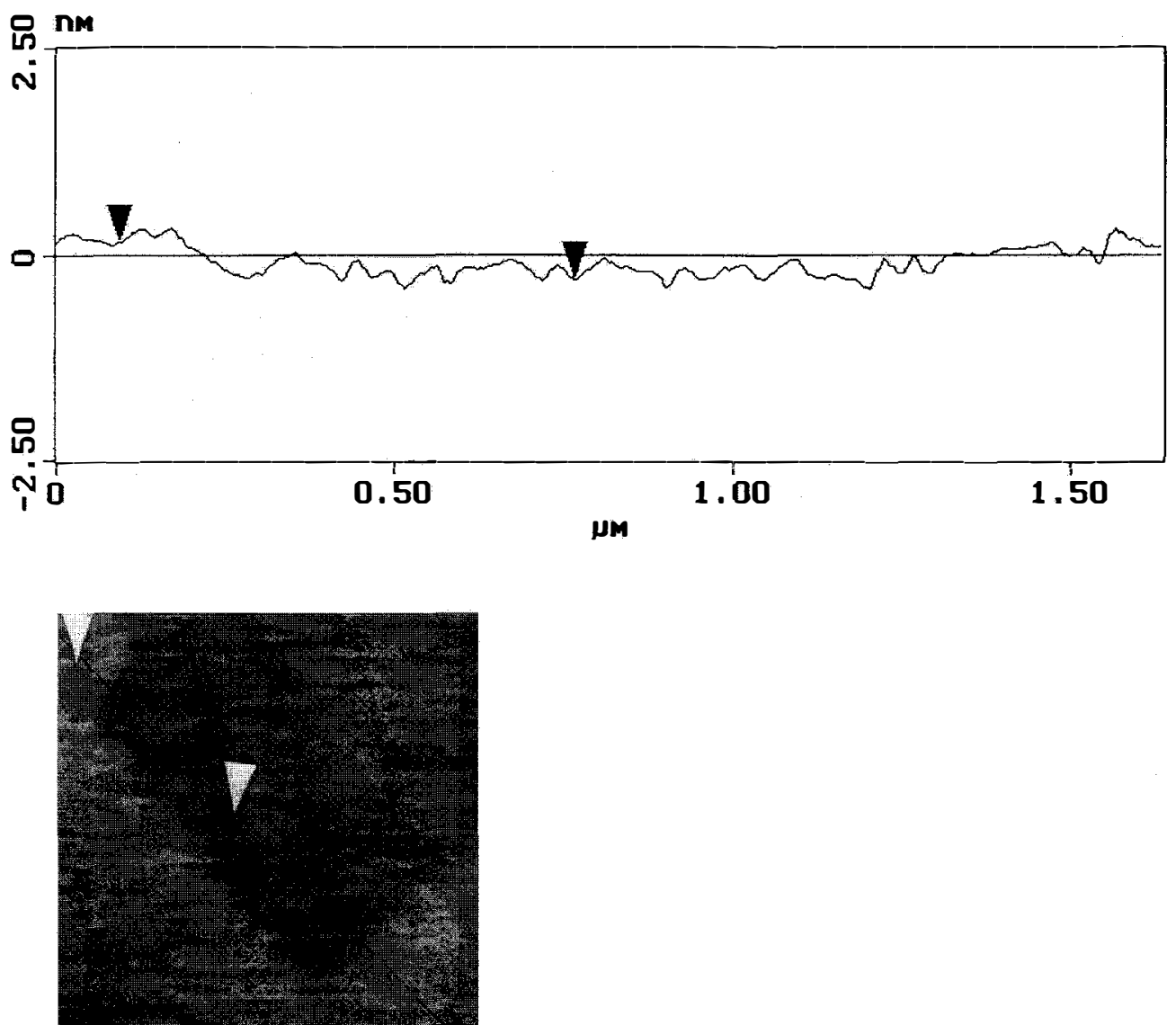

Fig. 5. AFM surface profile in the depressed area of the $2 \mathrm{H}-\mathrm{MoS}_{2}$ single crystal after the friction test. The vertical distance between the two cursors is $4.7 \AA$.

according to Amontons' law, Fig. 8(a). Furthermore, it is obvious that the surface is kept unaltered during AFM scans, Fig. 8(b). We correlate this observation with a strength of interlayer interaction higher in $\mathrm{MoO}_{3}$ than in $\mathrm{MoS}_{2}$ or $\mathrm{NbSe}_{2}$.

From our experiments it appears that when surfaces are unaltered during the measurements, as in the $\mathrm{MoO}_{3}$ case, friction behaviour on the nanometer scale could be compared to classical behaviour on the macroscopic scale.

During AFM friction measurements on $\mathrm{MoO}_{3}$, the linear evolution $F_{\mathrm{f}}$ vs. $F_{\mathrm{n}}$ follows the Amontons' law, which defines a constant coefficient of friction $\mu$. This law is verified when the true area of a contact is proportional to $F_{\mathrm{n}}$. This is often true on the macroscopic scale, where contact areas are rough. In this configuration, when $F_{\mathrm{n}}$ increases, new asperities are created by plastic and elastic deformations, increasing the true contact area. On the nanoscopic scale, a single elastic contact results in an $F_{\mathrm{f}} \propto F_{n}^{2 / 3}$ law [12]. We interpret our observation of a linear relationship $F_{\mathrm{f}}$ vs. $F_{\mathrm{n}}$ as evidence of the rough nature of the tip/surface contact. It allows us to extract a mean friction coefficient value of 0.022 for the investigated surface on the nanometer scale.

Although some phenomena, such as ploughing or surface deformation, act on the nanoscopic scale, they are negligible with respect to those acting at the macroscopic scale [2]. This explains 


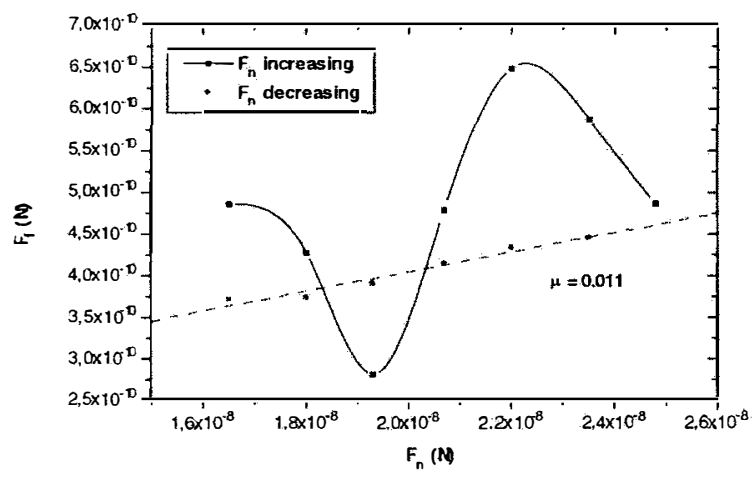

(a)

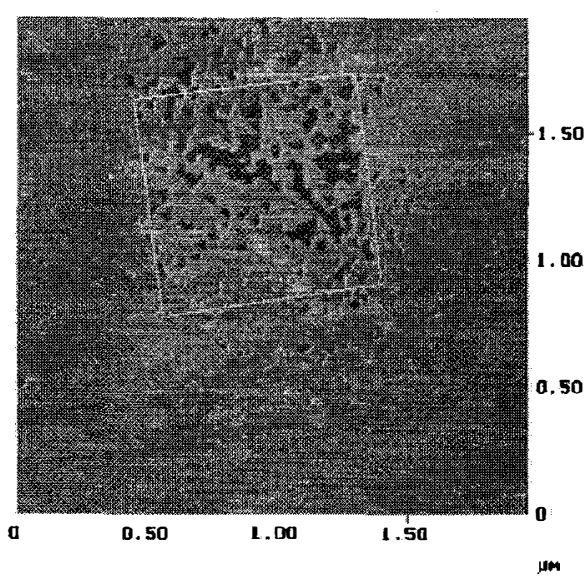

(b)

Fig. 6. (a) $F_{\mathrm{f}}$ vs. $F_{\mathrm{n}}$ during friction test on a $2 \mathrm{H}-\mathrm{NbSe}_{2}$ single crystal where measurements for increasing $F_{\mathrm{n}}$ are incoherent. A mean value $\mu=0.011$ is extracted for decreasing $F_{\mathrm{n}}$. (b) AFM picture recorded after the friction test, exhibiting a local wear of the surface.

why the nanoscopic values of $\mu$ are small compared to the macroscopic ones, as already observed for many materials $[19,23,24]$.

\section{Importance of parameter control}

The investigation of the frictional behaviour on the nanometer scale appears to be very delicate, and some parameters are susceptible to play a role of utmost importance. In particular, the question immediately arises of surface pollution in air. When this phenomenon is significant, friction measurements on the nanometer scale may be totally incoherent.

For instance, it is known that on silica there is an important condensation of water and other pollutants on the surface when kept in air. Following our experimental protocol, on an initially cleaned and dried silica surface, several force measurements at various times give different results, indicating that there is a growing layer of pollutants on the surface. Consequently, the tip/surface interface is continuously changing and force measurements remain impossible. When the surface is saturated by water, measurements are also impossible, because during a continuous scan the tip modifies the layer adsorbed on the surface.
With a lower scan speed, an interface equilibrium would be obtained, and coherent measurements performed.

If measurements are coherent in air (with $\mathrm{MoO}_{3}$ for instance), it does not mean that the surfaces are unpolluted, but that there is a dynamical equilibrium of the tip/surface interface including pollutant layer on the surface, pollutant meniscus around the tip, and material fragments pulled off from the surface and from the tip.

Obviously, in these conditions, friction measurements are not really characterising the investigated surface, but a system resulting from the adsorption of pollutants on the material surface.

As far as $\mathrm{MoO}_{3}$ is concerned, the linear dependence of $F_{\mathrm{f}}$ vs. $F_{\mathrm{n}}$ indicates the relatively low degree of pollution of the surface. Indeed, in the case of an important pollution, the capillary condensation of pollutants in contact asperities should induce a transition from a rough to a single asperity contact, and therefore to an $F_{n}^{2 / 3}$ dependence of friction [12].

Besides the importance of the thickness and of the dynamic stability of the pollution layer, we would like to define the role of two other parameters, the tip/surface interface composition and the scan speed, able to modify the value of $\mu$ for a given system. 

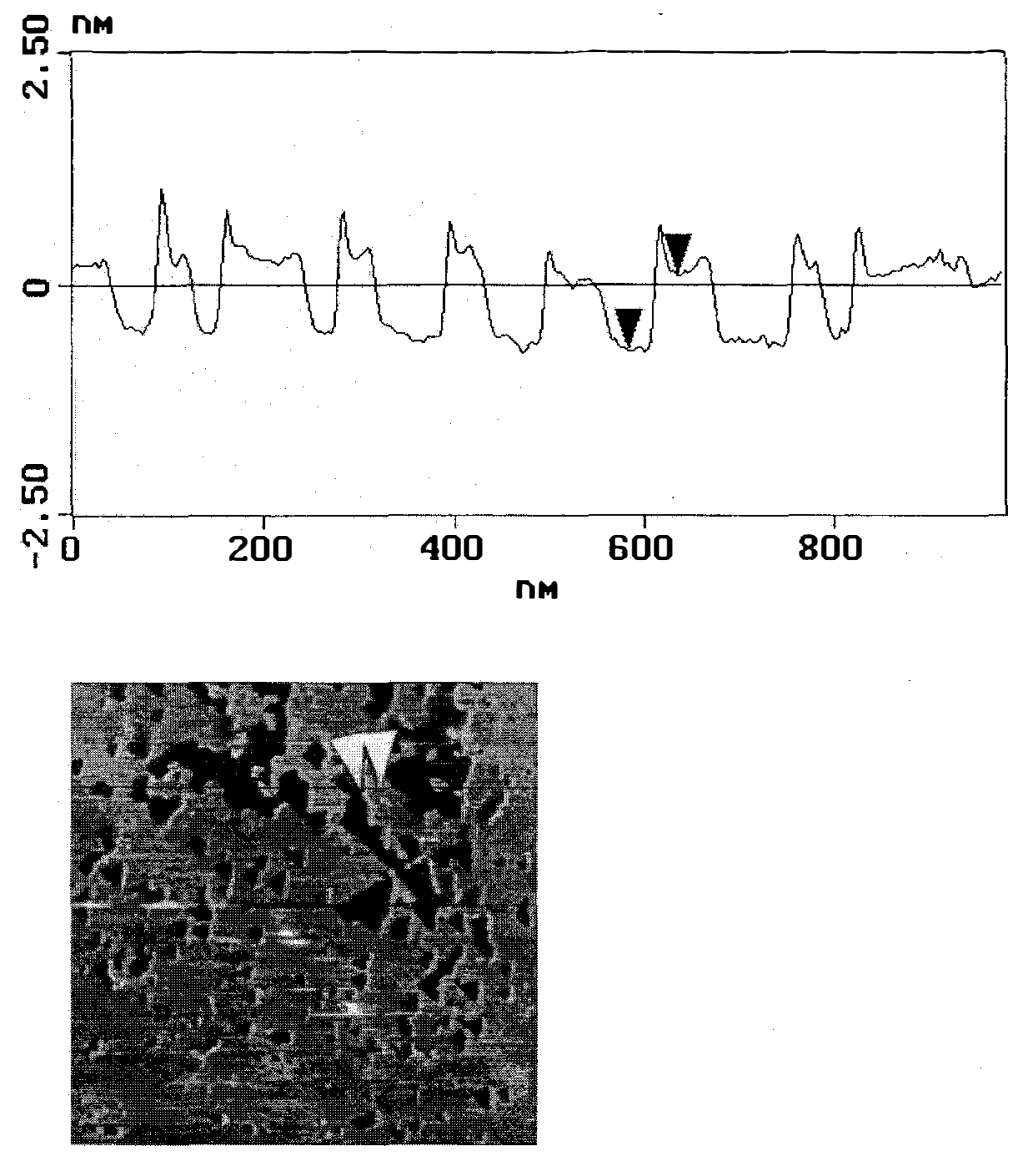

Fig. 7. AFM surface profile in the depressed area of the $2 \mathrm{H}-\mathrm{NbSe}_{2}$ single crystal after the friction test. The vertical distance between the two cursors is $8.1 \AA$.

We have realized friction experiments with $\mathrm{MoO}_{3}$ in argon, Fig. 9. Despite the expectation of a lower stage of pollution in argon, we observe a larger tip/surface adhesive interaction in our force measurements, which is generally due to a pollutant meniscus around the tip.

The linear dependence $F_{\mathrm{f}}$ vs. $F_{\mathrm{n}}$ is preserved, indicating that the thickness of the adsorbed layer cannot be responsible for the strengthening of the adhesive interaction.

Furthermore, we obtain a friction coefficient value of 0.014 , close to the $\mu$ value in air with respect to the uncertainty of measurements (see $k$ value in Section 3 ).

Therefore, despite the expectation of a lower adhesion of $F_{\mathrm{n}}$ due to a lower degree of pollution, at first glance there is no significant difference between studies performed in air and in argon.

Nevertheless, if the measurements are realized in a proof cell filled alternately with air and argon, large and reversible increases of the friction signal intensity are observed with argon (Fig. 10) due to modification of the composition of the tip/surface interface. Hence, it appears that, although there is no significant difference between studies performed in air and in argon, the modifications of the interface composition during experiments have a profound effect on friction measurements on this scale.

In another series of experiments carried out on $\mathrm{MoO}_{3}$, varying the scan speed is shown to induce variations of the friction signal for a constant 


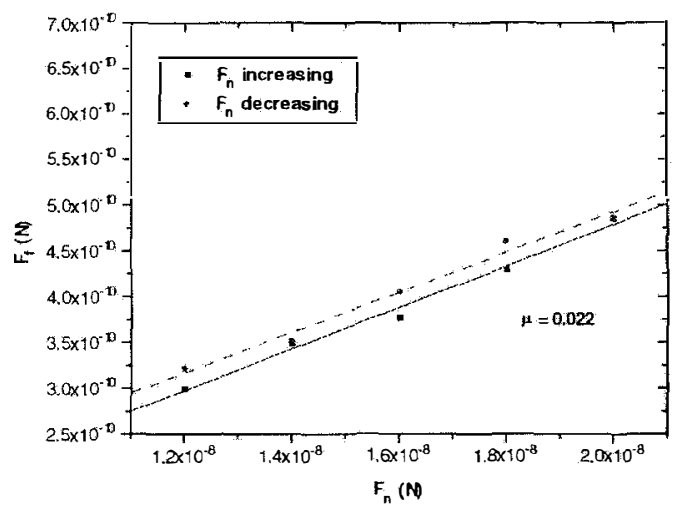

(a)

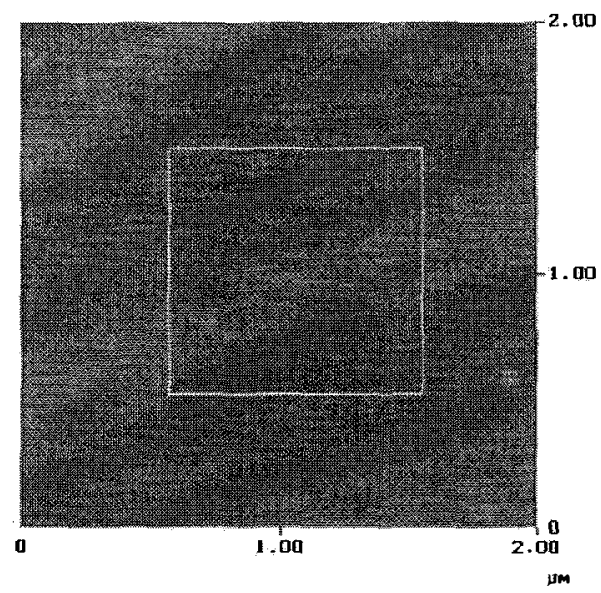

(b)

Fig. 8. (a) $F_{\mathrm{f}}$ vs. $F_{\mathrm{n}}$ during friction test on an $\alpha-\mathrm{MoO}_{3}$ single crystal. A mean value of $\mu=0.022$ is extracted. (b) AFM picture recorded after the friction test, showing no significant alteration of the surface.

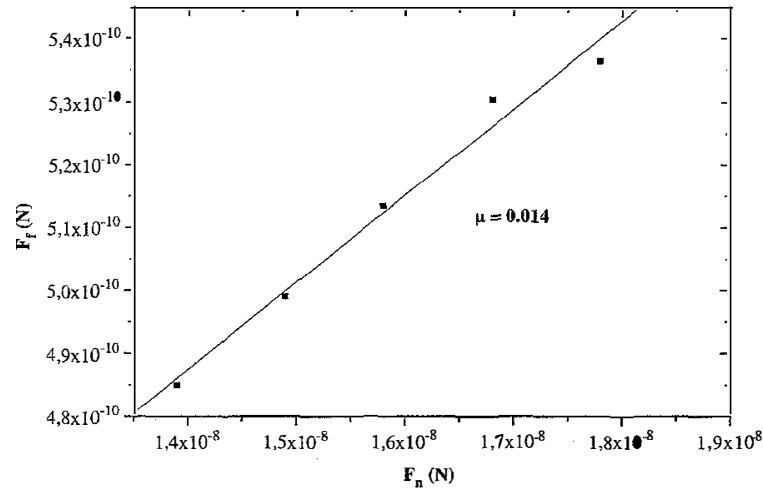

Fig. 9. $F_{\mathrm{f}}$ vs. $F_{\mathrm{n}}$ during friction test on an $\alpha-\mathrm{MoO}_{3}$ single crystal in an argon atmosphere. A mean value of $\mu=0.014$ is extracted.

value of $F_{\mathrm{n}}$ (Fig. 11), in discrepancy with Amontons' law (friction does not depend on sliding speed [10]), without variation of the topographic information (consistent pictures and constant RMS). Such a result could be explained by a modification of the tip/surface interface, and especially of the meniscus around the tip, related to the scan speed. Furthermore, before acquisition it is necessary to let the microscope scan for $5 \mathrm{~min}$ at least. During this period we can note a constant decrease of the friction signal, certainly due to a balancing of the sliding system.

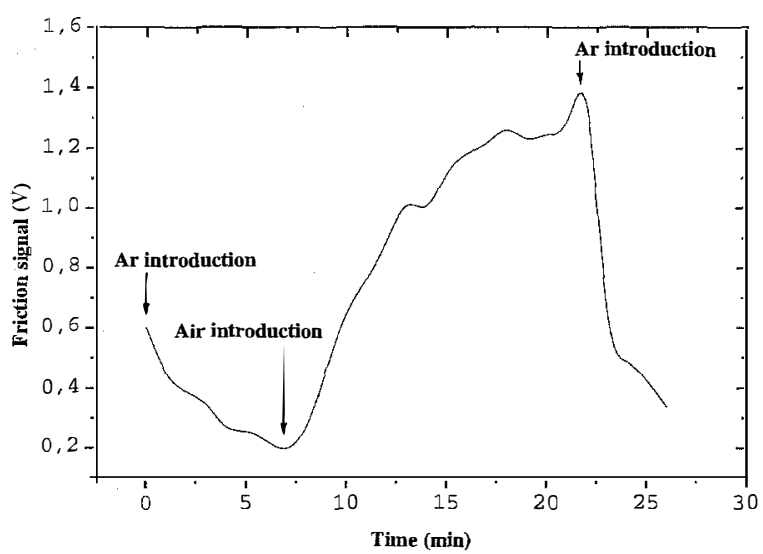

Fig. 10. Evolution of the friction signal for a constant value of $F_{\mathrm{n}}$ correlated to atmosphere changes during a test on an $\alpha-\mathrm{MoO}_{3}$ single crystal. The microscope is set in a proof cell filled alternately with argon and air.

\section{Conclusion}

Friction measurements on the nanometer scale have been performed with AFM. Some problems of an instrumental nature, such as cantilever and detector calibration, may occur and hinder us from obtaining reliable results. Also, depending on the nature of the investigated sample (roughness in particular), the surrounding atmosphere and the scanning parameters (scan speed for instance), the 


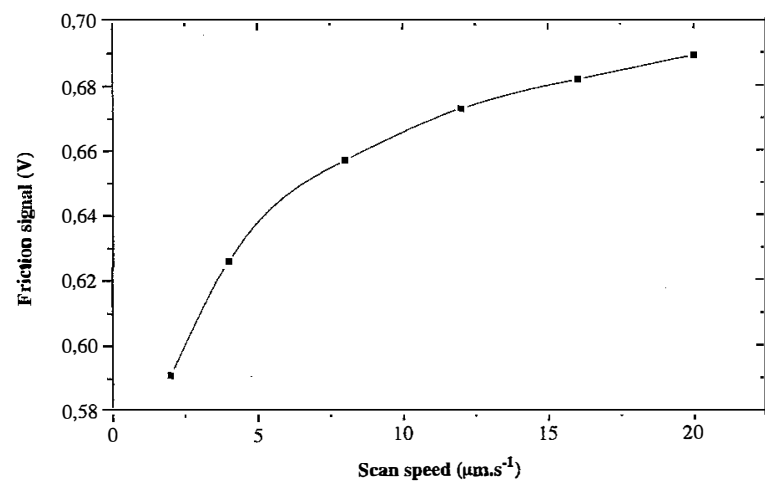

Fig. 11. Evolution of the friction signal for a constant value of $F_{\mathrm{n}}$ correlated to variation of scan speed during a test on an $\alpha-\mathrm{MoO}_{3}$ single crystal.

experimental results may or may not be meaningful. Therefore, well-defined experimental protocol and choice of parameters (e.g. atmosphere composition and scan speed) is absolutely necessary to overcome these unreliability difficulties. Under such conditions, there appears to be an accord between nanoscopic experimental results and macroscopic friction results predicted by models (Amontons' law for instance). As a matter of fact, some parameters of our experiments could be compared to those of macroscopic sliding. For instance, scan speed (a few micrometers per second) and strain due to the tip (about $200 \mathrm{MPa}$ calculated using the Hertz model) are close to parameters of fretting on the macroscopic scale. The interest of friction measurements with AFM is to focus on phenomena such as local wear, which are not accessible with macroscopic apparatus.

In the prototype surfaces investigated here, depending upon the layered system: $2 \mathrm{H}-\mathrm{MoS}_{2}$, $2 \mathrm{H}-\mathrm{NbSe}_{2}, \alpha-\mathrm{MoO}_{3}$, different behaviours such as plastic deformation, local wear or unalteration have been observed. Many phenomena related to friction measurements on this scale are not yet well understood. Nevertheless, upon offering an unrivalled way to discriminate rapidly between surfaces handled under real conditions and, in the absence of wear, to qualify them in terms of nanoscopic friction coefficients, AFM appears to be a promising tool for nanotribology in both laboratory and industrial environments.

\section{References}

[1] C.M. Mate, G.M. McClelland, R. Erlandsson, S. Shiang, Phys. Rev. Lett. 59 (17) (1987) 1942.

[2] M. Hirano, K. Shinjo, Phys. Rev. B 41 (17) (1990) 11837.

[3] W. Zhong, D. Tomanek, Phys. Rev. Lett. 64 (25) (1990) 3054.

[4] M. Hirano, K. Shinjo, R. Kaneko, Y. Murata, Phys. Rev. Lett. 67 (19) (1991) 2642.

[5] G.J. Germann, S.R. Cohen, G. Neubauer, G.M. McClelland, H. Seki, D. Coulman, J. Appl. Phys. 73 (1) (1993) 163.

[6] J. Krim, Scient. Am. 10 (1996) 48; Comm. Cond. Mater. Phys. 17(5) (1995) 263.

[7] B. Bhushan, J.N. Israelachvili, U. Landman, Nature 374 (13) (1995) 607.

[8] R. Nisman, P. Smith, G.J. Vansco, Langmuir 10 (1994) 1667.

[9] J. Kerssemakers, J.T.M. De Hosson, Appl. Phys. Lett. 67 (3) (1995) 347.

[10] D. Tabor, Surf. Sci. 2 (1974) 149.

[11] M. Bingelli, C.M. Mate, Appl. Phys. Lett. 65 (4) (1994) 415.

[12] C.M.J. Putman, M. Igarashi, R. Kaneko, Appl. Phys. Lett. 66 (23) (1995) 3221.

[13] J.A. Ruan, B. Bhushan, J. Appl. Phys. 76 (9) (1994) 5022.

[14] D.F. Ogletree, R.W. Carprick, M. Salmeron, Rev. Sci. Instrum. 67 (9) (1996) 3298.

[15] J.N. Israelachvili, Intermolecular and Surface Forces, 2nd edn, Academic Press, New York, 1992.

[16] J:M. Neumeister, W.A. Ducker, Rev. Sci. Instrum. 65 (8) (1994) 2527.

[17] J.E. Sader, Rev. Sci. Instrum. 66 (9) (1995) 4583.

[18] G.Y. Chen, R. Warmack, T. Thundat, D. Pallison, A. Huang, Rev. Sci. Instrum. 65 (8) (1994) 2532.

[19] J.A. Ruan, B. Bhushan, Trans. ASME 116 (1994) 378.

[20] F. Levy (Ed.), Physics and Chemistry of Materials with Layered Structures, vol. 2, Reidel, New York, 1976.

[21] J.A. Wilson, D. Yoffe, Adv. Phys. 18 (1969) 193.

[22] C.M. Lieber, Y. Kim, Thin Solid Films 206 (1991) 355.

[23] B. Bhushan, A.V. Kulkarni, V. Kointar, M. Boehm, L. Odoni, C. Martelet, M. Belin, Langmuir 11 (1995) 3189.

[24] J.A. Ruan, B. Bhushan, J. Appl. Phys. 76 (12) (1994) 8117. 